\title{
Level-Crossing Rate and Average Duration of Fades for TWDP Fading Channel
}

\author{
Ran Zhu, Junyi Yu, Zhinglong Jiang, Kun Yang*, Wei Chen
}

\begin{abstract}
This paper mainly proposes the Level-Crossing Rate (LCR) and Average Duration of Fades (ADF) of the Two Wave with Diffuse Power (TWDP) fading process. Both reference model and deterministic model are presented. The reference model is derived based on the description of TWDP process while the deterministic model is derived based on the Rice's sum-ofsinusoids (SOS). The derived deterministic model is proved to consist with the reference model based on the central limit theorem. The simulation results show an excellent agreement between these two models and the simulations.
\end{abstract}

Keywords-Fading channels; TWDP; Level-Crossing Rate; Deterministic simulation; Doppler frequency

\section{Introduction}

The Two Wave with Diffuse Power (TWDP) fading channel model, first proposed by Durgin [1], can be used to describe received signals consisted of two specular multipath waves with the diffuse components. This may occur for the typical narrow-band receiver, directional antennas and wideband signals which may increase the ratio of the specular to the diffuse power [1]. The envelope probability density functions (PDF) and its approximated form of TWDP fading model was also presented in [1]. Based on that, the BPSK system performance was analyzed by Soon H. Oh [2][3]. It points out the system performance was even worse under the TWDP channel condition than the Rayleigh channel condition when the amplitudes of two specular waves are equal and much larger than the diffuse power's [2].

Ran Zhu, Zhinglong Jiang

School of Information Engineering, Wuhan University of Technology China

Kun Yang

Ningbo Allmeas Co., Ltd \& Super Radio AS

Norway

Junyi Yu, Wei Chen

School of Automation, Wuhan University of Technology China
The performance of QAM system for this fading channel was also analyzed by Suraweera [4], Dixit [5] and Yao Lu [6], respectively, in which the results indicate that the system exhibits a poorer performance under the TWDP fading channel than the Rayleigh channel [6]. The statistics of TWDP fading model have also been studied in [7], where new expressions of the PDF and the cumulative distribution functions (CDF) are presented and these expressions can provide applicable results when the amplitudes of the two specular waves are much larger than the variance of the scattering components. Rao pointed out in [8] that the TWDP fading model can be regard as an extension of Rice fading model from the derivation of its PDF, in which the closedform expression for the Moment Generating Function of TWDP fading model is given. The expression of the LevelCrossing Rate (LCR) and Average Duration of Fades (ADF) for a special case that the incident angles of the two specular waves are perpendicular to the moving direction of receiver was also given in [8]. However, it needs to be pointed out that all the simulation parameters were obtained by using a stochastic method, the Monte Carlo Method. Besides, the higher order statistics of TWDP fading model still haven't been well investigated.

In this paper, the expressions of LCR and ADF for the TWDP fading model are given and verified with situations which show that the LCR and ADF are correlated to the Doppler frequencies of the two specular paths. If both of the two specular waves' Doppler frequencies approach to zero, the expression of its LCR can be simplified to its PDF multiplied by a constant, which was given in [8]. The simulation results of the LCR and ADF for the deterministic TWDP model is shown by using the Jakes power density. The LCR and ADF of the TWDP deterministic model is verified by the simulation results, and is also proved to be a good-fit for the reference model.

The rest of this paper is organized as follows. Section II derives the LCR and ADF of the TWDP reference model for both the general and special case. In Section III, the deterministic TWDP process is implemented by using the SOS method and parameterized by the method of exact Doppler spread (MEDS) under Jakes power spectral density [9]. The LCR and ADF for the deterministic TWDP model which is proved to match the reference model are derived from this deterministic process in Section IV. The comparisons between these two models and the simulation results are also given in this Section. Finally, the conclusions of this paper are drawn in Section V. 


\section{LCR and ADF Expression of The Reference Model for The TWDP Fading Channel}

\section{A. The derivation of $L C R$}

The description of TWDP channels can be denoted by the following equation:

$$
V=V_{1} \exp \left(j \phi_{1}\right)+V_{2} \exp \left(j \phi_{2}\right)+V_{d i f}
$$

where

$$
V_{d i f}=\mu_{1}+j \mu_{2}
$$

The phases of the two special waves $\phi_{1}, \phi_{2}$ are assumed to be independent from each other and uniformly distributed $\left(\phi_{1}, \phi_{2}\right.$ $\sim U(0,2 \pi))$. The envelopes of the two special waves $V_{1}, V_{2}$ are constant. The $\mu_{1}, \mu_{2}$ are independent zero-mean Gaussian random variables $\left(\mu_{1}, \mu_{2} \sim N\left(0, \sigma^{2}\right)\right)$ whose derivatives with respect to time are also independent Gaussian random variables with zero-mean, and independent from $\mu_{l}$ [9]. The variance can be expressed as:

$$
\operatorname{Var}\left(\dot{\mu}_{i}\right)=r_{\mu_{i} \mu_{i}}(0)=-r_{\ddot{\mu}_{i}}(0)=\beta_{i}
$$

By using the Jakes power spectral density [10], this formula is given by:

$$
\beta_{1}=\beta_{2}=\beta=2\left(\pi f_{\max } \sigma\right)^{2}
$$

The $f_{\max }$ represents the max Doppler frequency and the Doppler frequency of each specular component is $f_{l}$. The joint pdf of $\mu_{l}$ and its derivation can be expressed as follows:

$$
\begin{aligned}
& p_{\mu_{\rho_{1}} \mu_{\rho_{2}} \dot{\mu}_{\rho_{1}} \dot{\mu}_{\rho_{2}}}\left(x_{1}, x_{2}, \dot{x}_{1}, \dot{x}_{2}\right) \\
& =\frac{1}{2 \pi} \int_{-\pi}^{\pi} \frac{1}{2 \pi} \int_{-\pi}^{\pi} p_{\mu_{1}}\left(x_{1}-h_{1}\left(\phi_{1}, \phi_{2}\right)\right) \text {. } \\
& p_{\mu_{2}}\left(x_{2}-h_{2}\left(\phi_{1}, \phi_{2}\right)\right) \cdot p_{\mu_{1}}\left(\dot{x}_{1}+2 \pi s_{1}\left(\phi_{1}, \phi_{2}\right)\right) \text {. } \\
& p_{\dot{\mu}_{2}}\left(\dot{x}_{2}-2 \pi s_{2}\left(\phi_{1}, \phi_{2}\right)\right) d \phi_{2} d \phi_{1}
\end{aligned}
$$

where

$$
\begin{aligned}
& p_{\mu_{i}}\left(x_{i}\right)=\frac{1}{\sqrt{2 \pi} \sigma} \exp \left\{\frac{-x_{i}^{2}}{2 \sigma^{2}}\right\}, i=1,2 \\
& p_{\mu_{i}}\left(\dot{x}_{i}\right)=\frac{1}{\sqrt{2 \pi \beta}} \exp \left\{\frac{-\dot{x}_{i}^{2}}{2 \beta}\right\}, i=1,2
\end{aligned}
$$

$$
\begin{gathered}
h_{1}\left(\phi_{1}, \phi_{2}\right)=V_{1} \cos \phi_{1}+V_{2} \cos \phi_{2} \\
h_{2}\left(\phi_{1}, \phi_{2}\right)=V_{1} \sin \phi_{1}+V_{2} \sin \phi_{2} \\
s_{1}\left(\phi_{1}, \phi_{2}\right)=f_{1} V_{1} \sin \phi_{1}+f_{2} V_{2} \sin \phi_{2} \\
s_{2}\left(\phi_{1}, \phi_{2}\right)=f_{1} V_{1} \cos \phi_{1}+f_{2} V_{2} \cos \phi_{2}
\end{gathered}
$$

By transforming the Cartesian coordinates to the polar coordinates, the equation (5) can be expressed by [10]:

$$
\begin{aligned}
p_{\xi \theta \dot{\xi} \dot{\theta}}(z, \theta, \dot{z}, \dot{\theta})= & \frac{1}{z^{2}} p_{\mu_{\rho_{1}} \mu_{\rho_{2}} \mu_{\rho_{1}} \mu_{\rho_{2}}}(z \cos \theta, z \sin \theta, \dot{z} \\
& \cos \theta-\dot{\theta} z \sin \theta, \dot{z} \sin \theta+\dot{\theta} z \cos \theta)
\end{aligned}
$$

With the integrations as follows:

$$
p_{\xi \dot{\xi}}(z, \dot{z})=\int_{-\pi-\infty}^{\pi} \int_{-\infty}^{+\infty} p_{\xi \vartheta \dot{\xi}}(z, \theta, \dot{z}, \dot{\theta}) d \dot{\theta} d \theta
$$

LCR [9] can be obtained by using the following formula:

$$
N_{\xi}(r)=\int_{0}^{+\infty} \dot{z} p_{\xi \xi}(r, \dot{z}) d \dot{z}
$$

Further, the LCR of the reference model can be expressed as follows:

$$
\begin{aligned}
N_{\xi}(r)= & \int_{-\pi-\pi}^{\pi} \int^{\pi} \frac{r}{4 \pi^{2} \sigma^{2}} \cdot e^{-\frac{r^{2}+V_{1}^{2}+V_{2}^{2}}{2 \sigma^{2}}} \cdot e^{-\frac{V_{1} V_{2} \cos \left(\phi_{1}-\phi_{2}\right)+r h_{1}\left(\phi_{1}, \phi_{2}\right)}{\sigma^{2}}} . \\
& \left.\left(\begin{array}{l}
\sqrt{\frac{\beta}{2 \pi}} e^{-\frac{2 \pi^{2} s_{1}^{2}\left(\phi_{1}, \phi_{2}\right)}{\beta}}+ \\
\pi s_{1}\left(\phi_{1}, \phi_{2}\right) \cdot \operatorname{erf}\left(\sqrt{\frac{2}{\beta}} \pi s_{1}\left(\phi_{1}, \phi_{2}\right)\right.
\end{array}\right)\right)_{15 \phi_{2} d \phi_{1}}
\end{aligned}
$$

The results with the different Doppler frequencies of the two waves are shown in Figure.1, in which the LCR $N_{\xi}(\mathrm{r})$ is normalized by $f_{\max }$ in order to remove the influence of the max Doppler frequency.

It shows that the LCR of TWDP fading channel model has two peaks, and the LCR will increase with the increase of the two specular waves' Doppler frequencies $f_{1}, f_{2}$. 


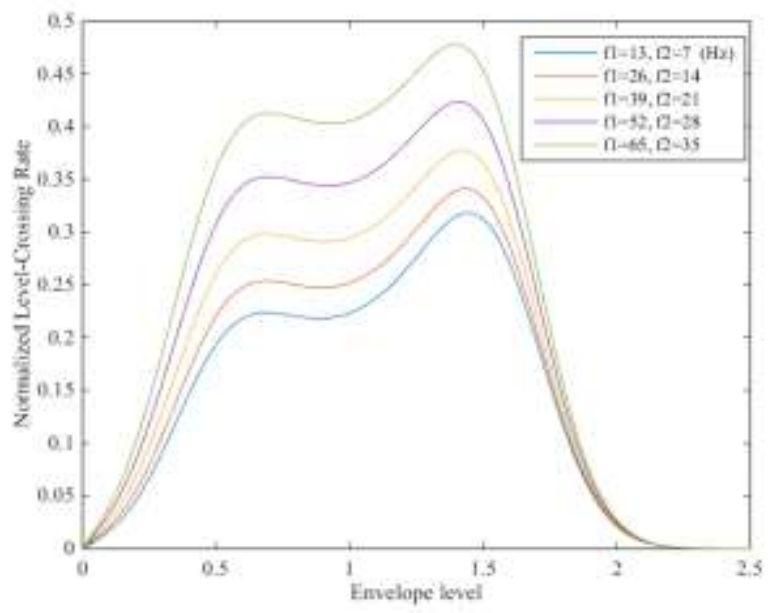

Figure.1. the normalized LCR $N_{\xi}(\mathrm{r}) / f_{\max }\left(V_{1}=1, V_{2}=0.6, \sigma=0.2, f_{\max }=91\right)$

\section{B. The LCR for The Special Case}

When the incident directions of the two specular components are perpendicular to the receiver moving direction, $f_{1}, f_{2}$ are equal to zero according to the Doppler frequency shift equation [9]. Thus, (15) can be simplified as:

$$
\begin{gathered}
N_{\xi}(r)=\sqrt{\frac{\beta}{2 \pi} \cdot \frac{r}{2 \pi \sigma^{2}} \cdot e^{-\frac{r^{2}+V_{1}^{2}+V_{2}^{2}}{2 \sigma^{2}}} \int_{-\pi}^{\pi} e^{\frac{V_{1} V_{2} \cos (\theta)}{\sigma^{2}}} .} \\
I_{0}\left(\frac{r \sqrt{V_{1}^{2}+V_{2}^{2}-2 V_{1} V_{2} \cos (\theta)}}{\sigma^{2}}\right) d \theta
\end{gathered}
$$

where $I_{0}(x)$ is zero order modified Bessel's function. The PDF of the TWDP model [1] is given in equation (17), which can be regarded as a part of the equation (16):

$$
\begin{aligned}
& f_{\text {TWDP }}(r)= \frac{r}{2 \pi \sigma^{2}} \cdot e^{-\frac{r^{2}+V_{1}^{2}+V_{2}^{2}}{2 \sigma^{2}}} \int_{-\pi}^{\pi} e^{\frac{V_{1} V_{2} \cos (\theta)}{\sigma^{2}}} . \\
& I_{0}\left(\frac{r \sqrt{V_{1}^{2}+V_{2}^{2}-2 V_{1} V_{2} \cos (\theta)}}{\sigma^{2}}\right) d \theta
\end{aligned}
$$

After that, the $N_{\xi}(\mathrm{r})$ can be obtained as follows:

$$
N_{\xi}(r)=\sqrt{\frac{\beta}{2 \pi}} \cdot f_{T W D P}(r)
$$

\section{The ADF}

The ADF [11] is defined as follows [9]:

$$
T_{\xi-}(r)=\frac{F_{\xi}(r)}{N_{\xi}(r)}
$$

where, $F_{\xi_{-}}(r)$ is the $\mathrm{CDF}$ of envelope $r$, which is the integration of the PDF. Thus, the CDF of TWDP model can be expressed as follows:

$$
F_{\xi_{-}}(r)=\int_{0}^{r} f_{T W D P}(r) d r
$$

It should be pointed out that the ADF can be only influenced by the LCR, since the CDF doesn't depend on the Doppler frequencies $f_{1}, f_{2}$. The ADFs of the reference model with different Doppler frequencies are shown in Figure.2.

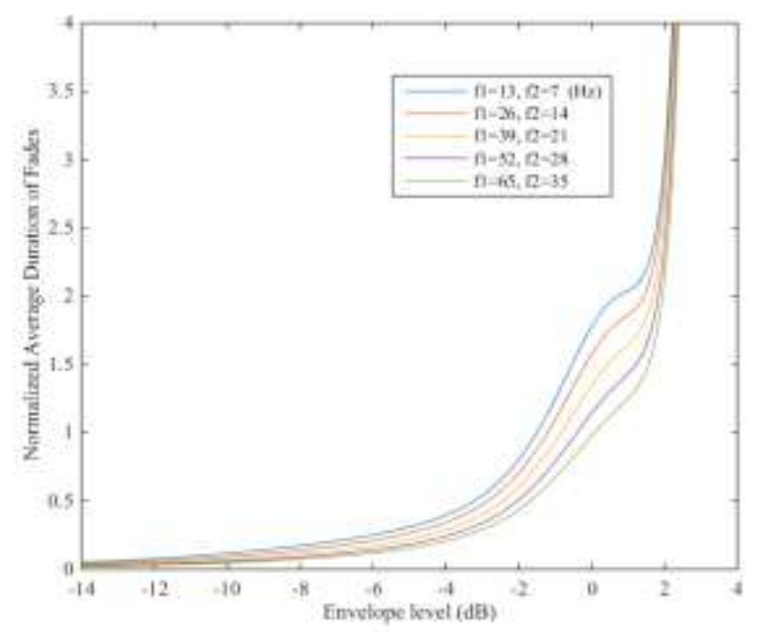

Figure. 2 the normalized $\operatorname{ADF} T_{\xi}(\mathrm{r}) \cdot f_{\max }\left(V_{1}=1, V_{2}=0.6, \sigma=0.2, f_{\max }=91\right)$

\section{The Deterministic Process of TWDP Channel}

From the TWDP channel model's description denoted by formula (1), the real part and imaginary part of the diffusion part are expressed by two independent zero-mean Gaussian random processes which can be modeled by using a superposition of a finite of sinusoids with given correlation properties based on the Rice's sum-of-sinusoids (SOS) [9]. According to the SOS theory, a stochastic Gaussian process $\mu_{i}(t)$ can be expressed by using an infinite SOS:

$$
\mu_{i}(t)=\lim _{N_{i} \rightarrow \infty} \sum_{n=1}^{N_{i}} c_{i, n} \cos \left(2 \pi f_{i, n} t+\theta_{i, n}\right), i=1,2
$$

If $N_{i}<\infty$ and all the model parameters $c_{i, n}, f_{i, n}$ and $\theta_{i, n}$ are realizations of random variables, the above stochastic model becomes a deterministic process denoted by: 
Proc. of the Fourth Intl. Conf. Advances in Computing, Communication and Information Technology- CCIT 2016 Copyright (C) Institute of Research Engineers and Doctors, USA .All rights reserved. ISBN: 978-1-63248-092-7 doi: 10.15224/ 978-1-63248-092-7-44

$$
\tilde{\mu}_{i}(t)=\sum_{n=1}^{N_{i}} c_{i, n} \cos \left(2 \pi f_{i, n} t+\theta_{i, n}\right), i=1,2
$$

where these parameters $c_{i, n}, f_{i, n}$ and $\theta_{i, n}$ can be computed by Method of Exact Doppler Spread (MEDS) [9]. This method is used because it can product an autocorrelation function which is quasi-optimal to given correlation properties like the Jakes power spectral density.

$$
\begin{gathered}
c_{i, n}=\sigma \sqrt{\frac{2}{N_{i}}}, i=1,2 \\
f_{i, n}=f_{\max } \sin \left[\frac{\pi}{2 N_{i}}\left(n-\frac{1}{2}\right)\right], i=1,2 \\
\vec{\theta}_{i}=\left(\theta_{i, 1}, \theta_{i, 2}, \ldots, \theta_{i, N_{i}}\right) \\
=\left(2 \pi \frac{1}{N_{i}+1}, 2 \pi \frac{2}{N_{i}+1}, \cdots, 2 \pi \frac{N_{i}}{N_{i}+1}\right), i=1,2
\end{gathered}
$$

Where, $N_{2}=N_{1}+1$ is implemented to make the real part and the imaginary part uncorrelated. The specular components can be parameterized as follows:

$$
V_{i} \cos \left(2 \pi f_{i} t+\theta_{v i}\right)+j V_{i} \sin \left(2 \pi f_{i} t+\theta_{v i}\right), i=1,2
$$

The Doppler frequencies $f_{1,} f_{2}$ are equal to zero for the special case since the incident directions of the two specular components are perpendicular to the receiver motion direction. However, if $f_{1}=f_{2}=0$, the $V_{1}, V_{2}$ can be regarded as one path, and the deterministic process turns out to be a Rice process. In order to avoid the above situation, $f_{1}$ can be set to zero, while $f_{2}$ can be equal to a very small value approaching to zero.

\section{Iv. The Exact Solution of The LCR and ADF for The Deterministic TWDP Process}

The time derivative of the deterministic Gaussian process (22) is given as follows:

$$
\dot{\tilde{\mu}}_{i}(\mathrm{t})=-2 \pi \sum_{n=1}^{N_{i}} c_{i, n} f_{i, n} \sin \left(2 \pi f_{i, n} t+\theta_{i, n}\right)
$$

The PDF of (22) and (27) can be expressed by using (28) and (29), respectively [12].

$$
\begin{aligned}
& \tilde{p}_{\mu_{i}}(x)=2 \int_{0}^{\infty} j_{h i}(v) \cos (2 \pi v x) d v \\
& \tilde{p}_{\mu_{i}}(\dot{x})=2 \int_{0}^{\infty} j_{s i}(v) \cos (2 \pi v \dot{x}) d v
\end{aligned}
$$

$$
\begin{gathered}
j_{h i}(v)=\prod_{n=1}^{N_{i}} J_{0}\left(2 \pi c_{i, n} v\right) \\
j_{s i}(v)=\prod_{n=1}^{N_{1}} J_{0}\left[(2 \pi)^{2} c_{i, n} f_{i, n} v\right]
\end{gathered}
$$

$J_{0}(x)$ is zero order Bessel's function here. $\mu_{1}, \mu_{2}$ and their corresponding derivatives are considered to be independent from each other [12]. Thus, their joint probability density function with the two specular components can be expressed by using a similar form as the reference model:

$$
\begin{aligned}
& \tilde{p}_{\mu_{1} \mu_{2} \dot{\mu}_{1} \mu_{2}}\left(x_{1}, x_{2}, \dot{x}_{1}, \dot{x}_{2}\right) \\
& =\frac{1}{4 \pi^{2}} \int_{-\pi}^{\pi} \int_{-\pi}^{\pi} \tilde{p}_{\mu_{1}}\left(x_{1}-h_{1}\left(\phi_{1}, \phi_{2}\right)\right) . \\
& \tilde{p}_{\mu_{2}}\left(x_{2}-h_{2}\left(\phi_{1}, \phi_{2}\right)\right) \cdot \\
& \tilde{p}_{\mu_{1}}\left(\dot{x}_{1}+2 \pi s_{1}\left(\phi_{1}, \phi_{2}\right)\right) \\
& \tilde{p}_{\mu_{2}}\left(\dot{x}_{2}-2 \pi s_{2}\left(\phi_{1}, \phi_{2}\right)\right) d \phi_{1} d \phi_{2}
\end{aligned}
$$

Similarly, the Cartesian coordinates can be transformed to polar coordinates as the derivation of reference model (12). After that, this joint PDF can be reformulated as follows:

$$
\begin{aligned}
& \tilde{p}_{\xi \xi}(z, \dot{z}) \\
& =\frac{2 z}{\pi^{2}} \int_{-\pi-\pi}^{\pi} \int_{-\pi}^{\pi} \int_{0}^{\pi} \int_{0}^{\infty} j_{h 1}(v) \cos [2 \pi v(z \cos \theta- \\
& \left.h_{1}\left(\phi_{1}, \phi_{2}\right)\right] d v \cdot \int_{0}^{\infty} j_{h 2}(v) \cos [2 \pi v(z \sin \theta- \\
& \left.\left.h_{2}\left(\phi_{1}, \phi_{2}\right)\right)\right] d v \cdot \int_{0}^{\infty} j_{s 1}(v \cos \theta) j_{s 2}(v \sin \theta) . \\
& \cos \left[2 \pi v\left(\dot{z}+s_{1}\left(\phi_{1}, \phi_{2}\right) \cos \theta-s_{2}\left(\phi_{1}, \phi_{2}\right) \sin \theta\right)\right] . \\
& d v d \phi_{1} d \phi_{2} d \theta
\end{aligned}
$$

According to (14), the LCR of the deterministic model can be derived as follows by using the joint PDF in (33):

$$
\begin{aligned}
& \tilde{N}_{\xi}(r) \\
& =\frac{2 r}{\pi^{2}} \int_{-\pi}^{\pi} \int_{-\pi}^{\pi} \int_{-\pi}^{\pi} \int_{0}^{\infty} j_{h 1}(v) \cos [2 \pi v(r \cos \theta- \\
& \left.\left.h_{1}\left(\phi_{1}, \phi_{2}\right)\right)\right] \cdot d v \int_{0}^{\infty} j_{h 2}(v) \cos [2 \pi v(z \sin \theta- \\
& \left.\left.h_{2}\left(\phi_{1}, \phi_{2}\right)\right)\right] d v \cdot \int_{0}^{\infty} j_{s 1}(v \cos \theta) j_{s 2}(v \sin \theta) . \\
& \dot{z} \cos \left[2 \pi v\left(\dot{z}+s_{1}\left(\phi_{1}, \phi_{2}\right) \cos \theta-s_{2}\left(\phi_{1}, \phi_{2}\right) \sin \theta\right)\right] . \\
& d v d \dot{z} d \phi_{1} d \phi_{2} d \theta
\end{aligned}
$$


In order to prove the correctness of above expression, a theoretical verification is given in the following context of this section. Assuming $N_{i}$ tends to infinity, the following equations can be given based on the central limit theorem [9].

$$
\begin{gathered}
j_{h 1}(v)=j_{h 2}(v)=e^{-2(\pi \tilde{\sigma} v)^{2}} \\
j_{s 1}(v \cos \theta) \cdot j_{s 2}(v \sin \theta)=e^{-2\left(\tilde{\beta}_{1} \cos ^{2} \theta+\tilde{\beta}_{2} \sin ^{2} \theta\right)(\pi v)^{2}} \\
\tilde{\sigma}^{2}=\lim _{N_{i} \rightarrow \infty} \sum_{n=1}^{N_{i}} \frac{c_{i, n}{ }^{2}}{2}=\sigma^{2} \\
\tilde{\beta}_{1}=\widetilde{\beta}_{2}=\lim _{N_{i} \rightarrow \infty} 2 \pi^{2} \sum_{n=1}^{N_{i}}\left(c_{i, n} f_{i, n}\right)^{2}=2\left(\pi f_{\max } \sigma\right)^{2}=\beta
\end{gathered}
$$

Combining (35), (36), (37) and (38) with (34), the following expression can be given:

$$
\begin{aligned}
& \lim _{N_{i} \rightarrow \infty} \tilde{N}_{\xi}(r) \\
& =\int_{-\pi-\pi}^{\pi} \int^{\pi} \frac{r}{4 \pi^{2} \sigma^{2}} \cdot e^{-\frac{r^{2}+V_{1}^{2}+V_{2}^{2}}{2 \sigma^{2}}} \cdot e^{-\frac{V_{1} V_{2} \cos \left(\phi_{1}-\phi_{2}\right)+r h_{1}\left(\phi_{1}, \phi_{2}\right)}{\sigma^{2}}} . \\
& \left(\begin{array}{l}
\sqrt{\frac{\beta}{2 \pi}} e^{-\frac{2 \pi^{2} s_{1}^{2}\left(\phi_{1}, \phi_{2}\right)}{\beta}}+ \\
\pi s_{1}\left(\phi_{1}, \phi_{2}\right) \cdot \operatorname{erf}\left(\sqrt{\frac{2}{\beta}} \pi s_{1}\left(\phi_{1}, \phi_{2}\right)\right)
\end{array}\right) d \phi_{1} d \phi_{2}
\end{aligned}
$$

it proves that the LCR expression of the deterministic model agrees with LCR expression of the reference model (15).

Since the CDF is the integral of its PDF, the CDF of the TWDP deterministic model (shown in Fig. 3) can be written as:

$$
\begin{aligned}
\tilde{F}_{\xi_{-}}(r)= & \frac{r}{2 \pi^{2}} \int_{0}^{\infty} \int_{0}^{\pi} j_{h 1}(v \cos \theta) j_{h 2}(v \sin \theta) d \theta . \\
& \int_{-\pi-\pi}^{\pi} \int_{-\pi}^{\pi} J_{1}(2 \pi r v) \cos \left(2 \pi v h_{1}\left(\phi_{1}, \phi_{2}\right)\right) d \phi_{1} d \phi_{2} d v
\end{aligned}
$$

According to the definition of ADF given in (19), the ADF can be calculated from the ratio of the LCR (34) and CDF (40). The LCR and ADF of the deterministic model, simulation and reference model are simulated by using MATLAB and shown in Figure.3, from which it can be found that the deterministic model, reference model and simulation agrees with each other perfectly.

For a special case that the $f_{1}$ is zero and the $\phi_{1}$ is set to be constant, the CDF and LCR of the deterministic model can be simplified as:

$$
\begin{gathered}
\tilde{F}_{\xi_{-}}(r)=\frac{r}{\pi} \int_{0}^{\infty} \int_{0}^{\pi} j_{h 1}(v \cos \theta) j_{h 2}(v \sin \theta) \int_{-\pi}^{\pi} J_{1}(2 \pi r v) \\
\cos \left[2 \pi v\left(V_{1} \cos \theta+V_{2} \cos \phi_{2}\right)\right] d \phi_{2} d v d \theta \\
\tilde{N}_{\xi}(r) \\
=\frac{4 r}{\pi} \int_{-\pi}^{\pi} \int_{-\pi}^{\pi} \int_{0}^{\infty} j_{h 1}(v) \cos \left[2 \pi v\left(r \cos \theta-h_{1}\left(\theta_{v 1}, \phi_{2}\right)\right)\right] d v \\
\int_{0}^{\infty} j_{h 2}(v) \cos \left[2 \pi v\left(r \sin \theta-h_{2}\left(\theta_{v 1}, \phi_{2}\right)\right)\right] d v d \phi_{2} . \\
\int_{0}^{\infty} \int_{0}^{\infty} j_{s 1}(v \cos \theta) j_{s 2}(v \sin \theta) \cdot \dot{z} \cos (2 \pi v \dot{z}) d v d \dot{z} d \theta
\end{gathered}
$$

Similarly, the simulation results for this special case are shown in Figure. 4. It needs to be mentioned that the LCR and ADF expression of the reference model can be found in (16).

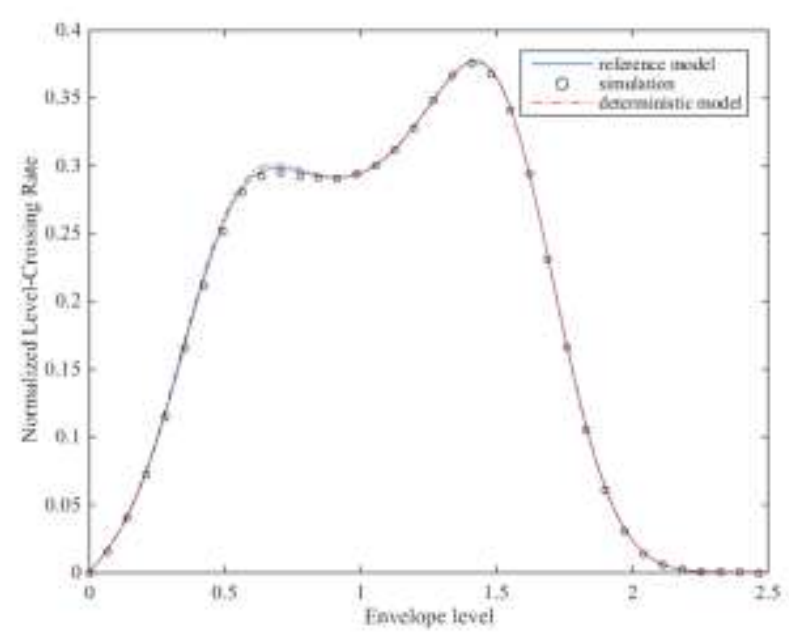

(a)

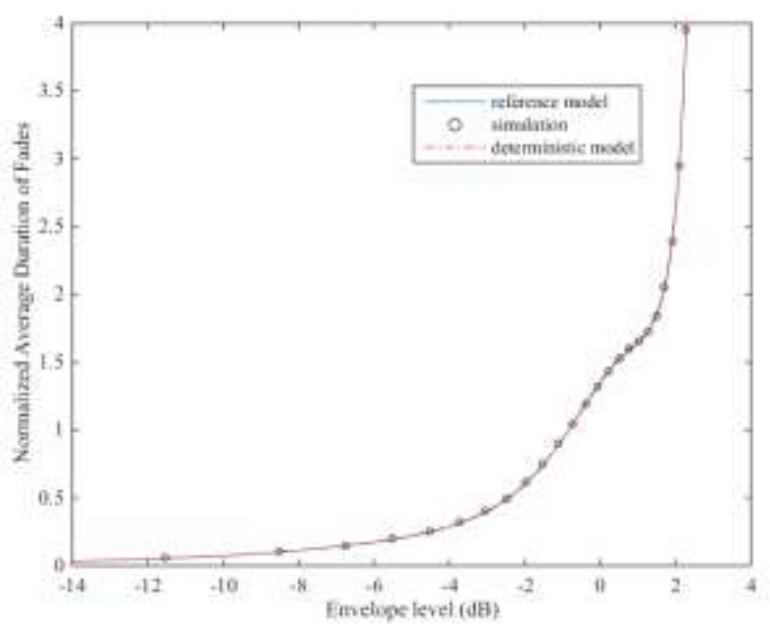

(b)

Figure.3. (a) the simulation results of normalized LCR $\left(N_{\xi}(\mathrm{r}) / f_{\max }\right)$ and (b) the simulations of normalized $\operatorname{ADF}\left(T_{\xi}(\mathrm{r}) \cdot f_{\max }\right)\left(V_{1}=1, V_{2}=0.6, \sigma=0.2, f_{\max }=\right.$ 91, $f_{1}=39, f_{2}=21, N_{1}=25, N_{2}=26, \theta_{\mathrm{v} 1}=\pi / 3, \theta_{\mathrm{v} 2}=\pi / 6$.) 


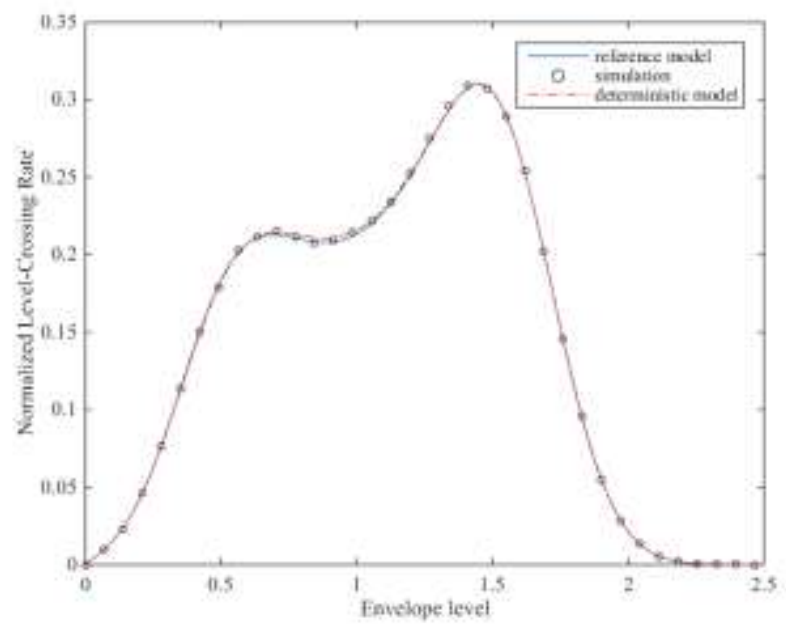

(a)

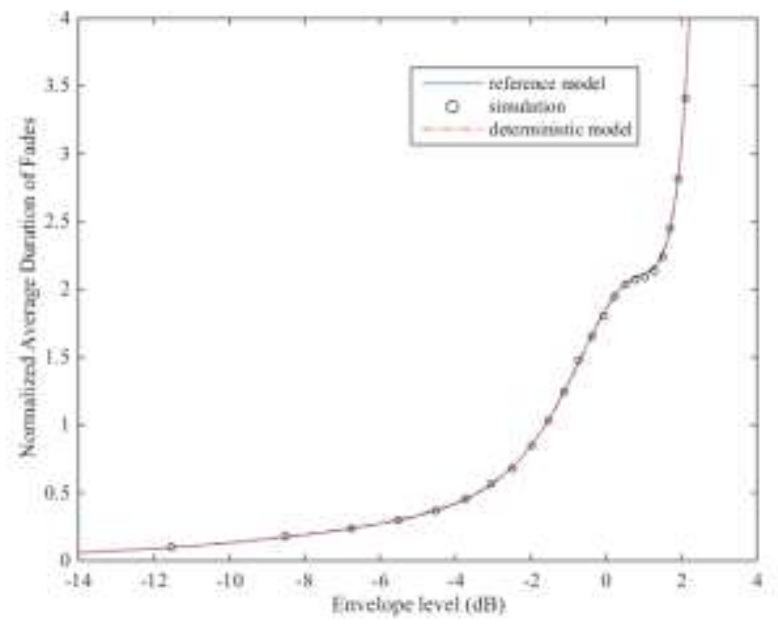

(b)

Figure.4. (a) the simulation results of normalized LCR $\left(N_{\xi}(\mathrm{r}) / f_{\max }\right)$ for the special case and (b) the simulation results of normalized $\mathrm{ADF}\left(T_{\xi}(\mathrm{r}) \cdot f_{\max }\right)$ for the special case $\left(V_{1}=1, V_{2}=0.6, \sigma=0.2, f_{\max }=91, N_{1}=25, N_{2}=26, \theta_{\mathrm{v} 1}=\right.$ $\pi / 3, \theta_{\mathrm{v} 2}=\pi / 6$.)

The simulation results of LCR and ADF expression of the reference model, deterministic model and simulation show perfect agreement in above figures for both general and special cases.

\section{v. Conclusion}

In this paper, the LCR and the ADF expressions of the reference model and the deterministic model for the TWDP fading process are derived for both general and special cases. The simulation results of the reference model shows the LCR will increase together with an increase of the frequencies, while the ADF will decrease. It indicates the values of the two specular waves' Doppler frequencies will result in influences on the higher order statistics of TWDP fading model. For the special case when the incident direction of the two specular paths are perpendicular to the receiver moving direction, the specular waves' Doppler frequencies turn out to be zero and the LCR expression becomes proportion to its corresponding PDF. Finally, the simulation results show that our LCR and ADF expression of the deterministic model matches with the reference model and simulation perfectly for both general and special case.

\section{References}

[1] G. D. Durgin, T. S. Rappaport, and D. A. de Wolf. "New analytical models and probability density functions for fading in wireless communications," IEEE Trans. Commun., vol. 50, no.6, pp. 1005- 1015, June 2002.

[2] S. H. Oh and K. H. Li, "BER performance of BPSK receivers over twowave with diffuse power fading channels," IEEE Trans. Wireless. Commun., vol. 4, no. 4, pp. 1448-1454, July 2005.

[3] S. H. Oh, K. H. Li, and W. S. Lee, "Performance of BPSK pre-detection MRC systems over two-wave with diffuse power fading channels," IEEE Trans. Wireless Commun., vol. 6, no. 8, pp. 2772-2775, Aug. 2007.

[4] H. A. Suraweera, W. S. Lee, and S. H. Oh, "Performance analysis of QAM in a two-wave with diffuse power fading environment," IEEE Commun. Lett., vol. 12, no. 2, pp. 109-111, Feb. 2008.

[5] D. Dixit and P. R. Sahu, "Performance of QAM Signaling over TWDP Fading Channels," IEEE Trans. Wireless Commun., vol. 12, no. 4, pp. 1794 - 1799, April 2013.

[6] Y. Lu and N. Yang, "Symbol error probability of QAM with MRC diversity in two-wave with diffuse power fading channels," IEEE Commun. Lett., vol. 15, no. 1, pp. 10-12, Jan. 2011.

[7] S. Saberali and N. Beaulieu, "New Expressions for TWDP Fading Statistics," IEEE Wireless Commun. Lett., vol. 2, no. 6, pp. 643-646, Dec. 2013.

[8] M. Rao, F. J. Lopez-Martinez and Andrea Goldsmith "Statistics and System Performance Metrics for the Two Wave With Diffuse Power Fading Model," 48th Annual Conference on Information Sciences and Systems (CISS), Princeton, NJ, pp. 1 - 6, March 2014.

[9] M. Pätzold, Mobile fading channels, 2nd ed. Chichester: Wiley, 2012.

[10] M. Pätzold, U. Killat and F. Laue, "An Extended Suzuki Model for Land Mobile Satellite Channels and Its Statistical Properties," IEEE Trans. on Vehicular Technology, vol. 47, no. 2, pp 617-630, May. 1998.

[11] S. O. Rice, "Statistical properties of a sine wave plus random noise," Bell Syst. Tech. J., vol. 27, pp. 109-157, Jan. 1948.

[12] M. Pätzold and C. A. Gutiérrez, "Level-Crossing Rate and Average Duration of Fades of the Envelope of a Sum-of-Cisoids," in Vehicular Technology Conference, 2008. VTC Spring 2008. IEEE, pp.488-494, 11-14 May 2008, doi: 10.1109/VETECS.2008.113 